\title{
Statics and Dynamics of V-shaped Micro-beams Under Axial Forces
}

\author{
Hassen M. Ouakad ${ }^{1}$, Nouha Alcheikh², Sofiane Ben Mbarek $^{2}$, Rodrigo Rocha², \\ Mohammad I. Younis ${ }^{2 *}$
}

${ }^{I}$ Mechanical and Industrial Engineering Department, College of Engineering, Sultan Qaboos University, PO-BOX 33, AlKhoudh, 123, Muscat, OMAN.

${ }^{2}$ Physical Science and Engineering Division, King Abdullah University of Science and Technology, Thuwal, 23955-6900, KSA.

*Corresponding Author: Mohammad.Younis@kaust.edu.sa

\begin{abstract}
This work proposes an examination into the static and dynamic behaviors of in-plane V-shaped microbeam under both electric forces and axial loads. The microbeams are actuated with two separate electrodes of uniform air-gap across their length. The effects of the initial rise and DC bias voltage are examined while varying the axial loads ranging from compressive to tensile. The numerical analysis is based on a nonlinear equation of motion of a shallow V-shaped microbeam. The static and eigenvalue problem were solved using a modal expansion based reduced-order modeling for numerous equilibrium positions. The analytical model is validated by comparing to an experimental case study. The results show rich and diverse static and dynamic behavior. It is shown that the microbeam may exhibit only the pull-in or snap-through and pull-in instabilities. Various multi-state and hysterics behaviors are demonstrated when varying the actuation forces and the initial rise. High tunability is demonstrated when varying the axial and DC loads for the first two symmetric vibration modes. With various axial load and DC actuation options and different geometrical configurations, this particular V-shaped show a capacity of increasing the static deflection range before pull-in, allowing more variation of its fundamental natural frequency. Therefore, it could be more promising for the realization of different wide-range tunable micro-resonator as compared to the regular straight and even bell-shaped micro-beams. These results are very useful in micro-scale applications that can be benefit for designing some structures with low power consumption, high sensitivity, and wide tuning range. Such rich behavior can be very useful for high performance micro-scale applications designs.
\end{abstract}

Keywords: V-shaped micro-beam; Electrostatic actuation; Axial forces; Pull-in and snap-through instabilities; Dynamic behavior; 


\section{Introduction:}

Microelectromechanical system (MEMS) based curved micro-beams are attracting substantial interests for actuating and sensing applications, owing to their high sensitivities, high resolution, low power consumption, high performance, and light weight [1-5]. Among the various actuation methods, electrostatic and electrothermal actuations are the two most effective mechanisms for achieving in-plane motion. Despite the high driving voltage, electrostatic excitation requires low power consumption, high speed, and short response time [6, 7]. Owing to these advantages, they have been used in wide range of applications, such as micro-switch and pressure sensors [8]. On the other hand, the electrothermal actuation, despite the high-power consumption, has been shown to be more robust and requires only low actuation voltages $[9,10]$.

One of the most important effects considered in electrostatically actuated curved micro-beams are pull-in and snap-through (bi-stable) instabilities [11]. The pull-in instability occurs when the applied voltage passes a critical value, and may lead to the failure of the micro-beams [12]. Bistability occurs when at the same voltage; a curved micro-beam exhibits a transition between the two stable states [13]. Various shapes of curved micro-beams have been used to generate inplane motions, such as Arc-shaped [14], Cosine-shaped [15-18], and V-shaped [16]. The pull-in and bi-stable behaviors of the in-plane Arches micro-beams (Arc- and Cosine-shaped) have been widely studied and investigated for various applications, such as micro-switches, filtering, microvalves, logics, mechanical memories, pressure sensors, and energy harvesting [3, 20, 21]. Also, the arch micro-resonators have received considerable attention in the literature to examine their static and dynamic behaviors [22]. The static behaviors of electrostatically actuated in-plane Vshaped structures, on the other hand, have attracted significant attention in recent years, as they have been proven to be fast and yield large output displacement [23]. However, few studies have focused on studying their dynamical behavior.

In the literature, the static and dynamic behaviors of arches micro-beams under axial forces are well investigated [21]. Practically, such an axial load can be induced through various techniques, for instance through passing an electrical current inducing Joule's heating $[15,16]$, or through having external actuation mechanism, for example a side electrostatic electrode [17, 18]. These studies have proven their ability for various applications in sensing, filtering, and memory [24]. In previous works, experimental and analytical investigations were presented for the static and dynamic behavior of arches micro-resonators under electrostatic actuation and electrothermal actuation (compressive axial forces) [15]. In [3], the possibilities of mode veering and crossing were examined and application for pressure sensors was proposed. Also, the static and dynamics of V-shaped micro-resonators under electrothermal excitation have been investigated in [25]. However, pull-in and snap-through of electrostatically actuated V-shaped micro-beam and their dynamic behavior under axial forces remain un-adequately explored. It is known that the resonance frequency of the arch micro-resonators is highly dependent on geometrical parameters, axial loads, and actuation forces. In sensors applications, the sensitivity and the dynamic ranges are essential parameters. Therefore, it is important to investigate and examine the dynamic response of the $\mathrm{V}$-shaped micro-resonators as varying the input axial forces and geometrical parameters. 
This study is mainly to examine in depth the effect electrostatic actuation superimposed to of axial loads on the static, pull-in and bi-stable behaviors of in plane clamped-clamped V-shaped micro-beams sandwiched between two electrodes with uniform and constant air-gap across the length. Also, their dynamic responses and natural frequencies of the first two symmetric modes under various initial rises are studied in details. An analytical model is developed, based on multi-mode Galerkin-based reduced-order model to solve the static and eigenvalue problems under various electrostatic actuation scenarios and axial forces. The model is validated by comparing with an experimental result of a case study. We prove a wide range tunability and flexibility of shifting the pull-in or bi-stable behaviors when controlling axial forces ranging from compressive loads to tensile loads. The results show that the dynamics of $\mathrm{V}$-shaped microbeam are very sensitive to axial loads, which can be helpful for sensors applications, such as pressure and magnetic sensors. On the other hand, the analytical model can be used as a guideline for designing micro-sensors and micro-actuators with low power consumption, high sensitivity, high displacement, high stable range, and wide tuning range of electrostatically actuated MEMS.

\section{Problem Formulation:}

In the below, the mechanical and geometrical equations of $\mathrm{V}$-shaped microbeam, Fig. 1, under the effect of both axial loads and electric forces are presented. The V-shaped beam is clampedclamped from both sides at its respective left and right anchors and is made from Silicon on insulator wafer layer with $\rho$ as its material mass density and $E$ its respective Young's modulus of elasticity. It possesses the following geometrical properties: $L$ (Length), $b$ (width) and $h$ (thickness). Consequently, $A=b h$ and $I=b h^{3} / 12$ are respectively the cross-sectional area and second moment of inertia of the rectangular $\mathrm{V}$-shaped microbeam.

As numerous mathematical fitting equations can be assumed to designate the initial V-shape (profile) of the investigated microbeam [26, 27]; we assume in this work the below expression showing an excellent agreement with the 3D Optical profilometer images of the investigated structures:

$$
w_{0}(x)= \begin{cases}b_{0}\left[\frac{x}{L / 2}-\frac{1}{\Delta\left(b_{0}\right)} \operatorname{Sin}\left(2 \pi \frac{x}{L / 2}\right)\right] ; & \text { for } 0 \leq x \leq L / 2 \\ 2 b_{0}-b_{0}\left[\frac{x}{L / 2}-\frac{1}{\Delta\left(b_{0}\right)} \operatorname{Sin}\left(2 \pi \frac{x}{L / 2}\right)\right] ; & \text { for } L / 2 \leq x \leq L\end{cases}
$$

In the above, $b_{0}$ and $\Delta\left(b_{0}\right)$ both designate the initial rise at the mid-point and a detuning parameter function of the maximum rise, respectively, mainly used for numerical fitting of the actual microbeam profile. 


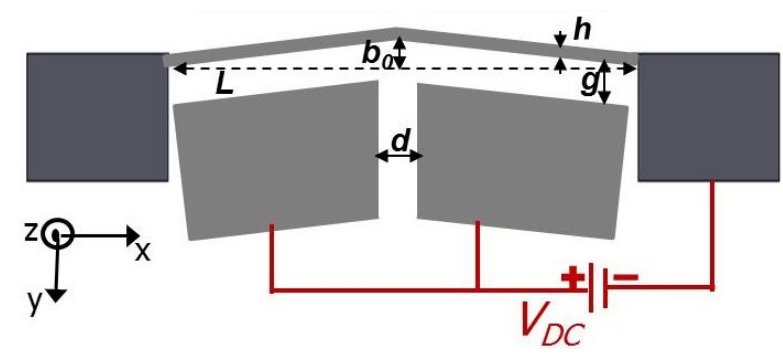

(a)

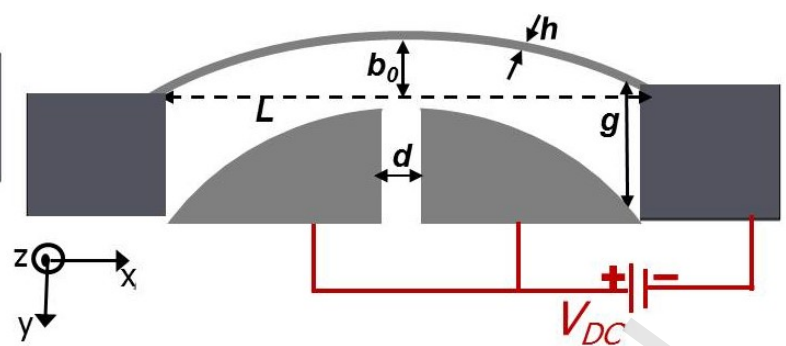

(b)

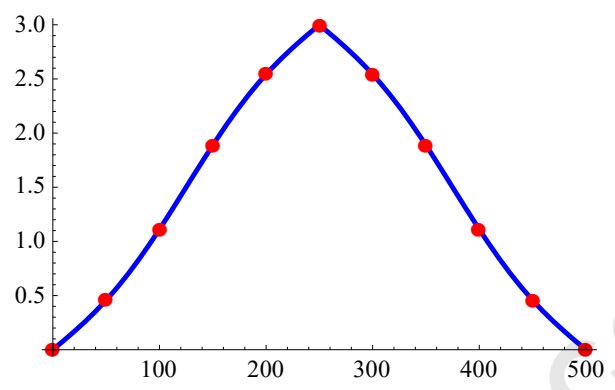

(c)

Fig. 1. Schematic of an electrostatically actuated clamped-clamped (a) V-shaped and (b) Arc-shaped micro-beams resonators, and (c) comparison with the fitting equation (1), continuous line with the 3D Optical profilometer imaged profile, red dots.

A static DC voltage $V_{D C}$ is applied between the movable $\mathrm{V}$-shaped and its stationary actuating electrodes resulting into an electrostatic force that is uniform and equal across the length, like the case of a straight beam. This attractive force pushes the $\mathrm{V}$-shaped electrode to be attracted in the direction of the stationary actuating electrodes and therefore resulting into a change in the structure effective stiffness. Assuming a nonlinear model based on the so-called Euler-Bernoulli beam theory, the mechanical behavior (in-plane deflection) of the V-shaped microbeam and respective boundary conditions are governed by the following partial-differential equation:

$$
\left\{\begin{array}{c}
E I \frac{\partial^{4} w(x, t)}{\partial x^{4}}+\rho A \frac{\partial^{2} w(x, t)}{\partial t^{2}}+\tilde{c} \frac{\partial w(x, t)}{\partial t}=N_{\text {total }}\left(\frac{\partial^{2} w(x, t)}{\partial x^{2}}-\frac{d^{2} w_{0}(x)}{d x^{2}}\right)+F_{e}(w(x, t)) \\
\left.w(x @ 0)\right|_{\forall t}=\left.w(x @ L)\right|_{\forall t}=0 ;\left.\quad \frac{\partial w(x @ 0)}{\partial x}\right|_{\forall t}=\left.\frac{\partial w(x @ L)}{\partial x}\right|_{\forall t}=0
\end{array} ;\right.
$$

In the above, $w(x, t)$ symbolizes the microbeam in-plane displacement in the $y$-direction, $\tilde{c}$ represents the overall viscous damping coefficient, and $F_{e}$ represents the resultant electric load without taking into account the fringing-fields effect. It is worth mentioning that we have examined the electric fringing-fields effect and found it of negligible outcome. Moreover, $N_{\text {total }}$ is the entire resultant axial force and can be written as follows:

$$
N_{\text {total }}=\left(N_{a x}+\frac{E A}{2 L} \int_{0}^{L}\left[w^{\prime 2}-2 w^{\prime} w_{0}^{\prime}\right] d x\right) ;
$$


In Eq. (3), $N_{a x}=\sigma_{a x} A$ symbolizes the microbeam axial load and $\sigma_{a x}$ is the residual axial stress.

The symbol (.) $)^{\prime}$ denotes the first rate of change of the function with respect to the variable space $x$. The $2^{\text {nd }}$ term in Eq. (3) represents the resultant nonlinear force to model both the mid-plane stretching and initial curvature effects.

Assuming a parallel-plates theory, the resultant electrostatic force, which has mainly a spacedependent profile, can be written as follows:

$$
F_{e}(w(x, t))=\frac{\varepsilon_{0} b V_{D C}^{2}}{2(g-w(x, t))}[1-U(x-L / 2+d / 2)+U(x-L / 2-d / 2)]
$$

where $V_{D C}$ is an assumed DC load applied between the microbeam and one of its stationary electrodes, $\varepsilon_{0}$ is the permittivity of the air, $g$ represents the initial gap size between the microbeam and the electrodes, $d$ stands for the lateral shift between the left and right stationary electrodes, $U(x)$ represents the unit step function.

The above electrostatic force expression is accordingly substituted into Equation (2) and formerly the resultant equation is mathematically discretized using the modal expansion technique [28]. For this, the $\mathrm{V}$-shape microbeam deflection is expanded as follows:

$$
w(x, t)=\sum_{k=1}^{N} \psi_{k}(x) q_{k}(t)
$$

In the above Eq. (5), the functions $\psi_{j}(1 \leq j \leq N)$ represent the mode-shapes of an un-forced and un-damped doubly-clamped straight beam and $q_{k}(t)(1 \leq k \leq N)$ are their respective timedependent modal coordinate amplitude which will form a reduced-order model consisting of Ndifferential equations and are to be numerically simulated [29]. The derived ROM can be used to calculate the microbeam natural frequencies.

The static deflection is first calculated numerically by setting all time dependent terms in the ROM differential equations equal to zero [29]. Then the modal amplitudes $q_{k}(t)$ are replaced by unknown constant quantities $a_{i}$. This results in a system of nonlinear algebraic equations in terms of those coefficients. The system is then solved numerically using the Newton-Raphson method. Each equilibrium point can be stable or unstable and this query of stability is very important for any dynamical systems [29]. Before proceeding further, it should be noted that the stability analysis in the below would be a local one because the original nonlinear system will be next linearized to compute the eigenvalue problem [29]. Formerly, the eigenvalue problem is solved to get the proper microbeam natural frequencies and this through numerically computing the eigenvalues of the Jacobian matrix of the generated ROM [29]. For this, we consider the ROM modal amplitudes discretized equations, which can be written in a state-space form as: 


$$
\dot{W}=R(W)
$$

where

$$
W=\left[q_{1}, q_{2}, \ldots, q_{n}\right]^{T},
$$

is the $\mathrm{V}$-shaped resonator normalized modal coordinates vector and $R(W)$ is a right-hand side vector representing the stiffness coefficients. Note that the vector $R(W)$ is a nonlinear function of the modal amplitudes' functions $q_{k}(t)$. We considered in this particular problem the symmetric and anti-symmetric mode-shapes in the ROM to get all the possible natural frequencies of the actuator. Next, we split $W$ into a static part $w_{s}$, representing the equilibrium position due to the $\mathrm{DC}$ actuation, and a dynamic part $\eta(t)$ representing the dynamic perturbation around the equilibrium position, that is:

$$
W=w_{s}+\eta(t),
$$

Then, substituting Eq. (8) into Eq. (6), using a Taylor series expansion assuming small perturbation $\eta(t)$, then eliminating the higher-order components, and using the fact that $R\left(w_{s}\right)=0$, we get the following equation:

$$
\dot{\eta}(t)=J\left(w_{s}\right) \eta(t),
$$

where $J\left(w_{s}\right)$ represents the Jacobian matrix of the actuator evaluated at its equilibrium points [29]. To get the natural frequencies of the actuator at a given DC voltage, we substitute the static solution $w_{s}$ into the matrix $J$ and then find its corresponding eigenvalues which are calculated by solving numerically the below characteristic equation for the eigenvalue $\lambda$, that is:

$$
\operatorname{det}\left(J\left(w_{s}\right)-\lambda I\right)=0,
$$

where the matrix $I$ represents the identity matrix and "det" refers to the determinant operator. Then each individual calculated eigenvalue represents a particular natural frequency of the actuator. In general, $\lambda$ is a complex number and if its real part is positive then the respective equilibrium point is unstable, and in contrary if its real part is negative therefore the respective equilibrium point is stable. 


\section{Static Analysis:}

As a case study, we consider the V-shaped micro-beam in Table 1 with the summarized dimensions and material properties. A convergence examination was first implemented and five modes was found to be satisfactory for numerical convergence $(N=5)$.

Table 1: V-SHAPED MICROBEAM (MATERIAL and GEOMETRICAL PROPERTIES of DOPED SINGLE-CRYSTAL SICLICON).

\begin{tabular}{|l|c|l|c|}
\hline Properties & Value & \multicolumn{1}{|c|}{ Properties } & Value \\
\hline Mass density $\rho\left(\mathrm{kg} / \mathrm{m}^{\mathbf{3}}\right)$ & 2332 & Initial rise $\boldsymbol{b}_{\mathbf{0}}(\boldsymbol{\mu m})$ & vary \\
\hline Effective Young's modulus $E(\boldsymbol{G P a})$ & 136 & Vertical gap size $\boldsymbol{g}(\boldsymbol{\mu m})$ & 8 \\
\hline Poisson's ratio & 0.28 & $\begin{array}{l}\text { Coefficient of thermal expansion } \alpha \\
\left({ }^{\circ} C^{-1}\right)\end{array}$ & $2.6 \times 10^{-6}$ \\
\hline Length $\boldsymbol{L}(\boldsymbol{\mu m})$ & 500 & Electrical resistivity $\rho_{\mathrm{e}}(\mathbf{\Omega} . \mathbf{m})$ & $1.53 \times 10^{-4}$ \\
\hline Thickness $\boldsymbol{h}(\boldsymbol{\mu m})$ & 2 & $\begin{array}{l}\text { Thermal conductivity coefficient } k \\
(\mathbf{W} / \mathbf{m K})\end{array}$ & 300 \\
\hline Width $\boldsymbol{b}(\boldsymbol{\mu m})$ & 25 & $\begin{array}{l}\text { Convective heat transfer coefficient } \boldsymbol{h}_{\boldsymbol{c}} \\
\left(\mathbf{W} / \mathbf{m}^{\mathbf{2}} \mathbf{K}\right)\end{array}$ & 35 \\
\hline
\end{tabular}

Fig. 2a shows the maximum static displacement of the $\mathrm{V}$-shaped micro-beam for various values of normalized axial force load $N$ and $V_{D C}=0 \mathrm{~V}$, where $N=N_{a x} L^{2} / E I$. It is worth noting that in Fig. 2a, the deflection is measured relatively from the initial profile of the $\mathrm{V}$-shaped structure assuming zero axial force and zero DC load, Fig. 2c. A positive relative displacement indicates a downward deflection of the micro-beam toward the stationary actuating electrodes. Additionally, a positive value of the axial force stands for a tensile load and negative value means a compressive load. Fig. 2a shows as well the effect of tensile and compressive axial forces with zero DC voltage load. The respective static profiles for negative, zero and positive axial loads are shown in Figs. 2b, 2c and 2d, respectively. It is clear from the profiles that an assumed positive axial load reduces the curvature of the V-shaped micro-beam pushing it toward the lower actuating electrodes. In contrary, any compressive axial loads enhance further the bending of the $\mathrm{V}$-shaped microbeam pushing it up and away for the stationary electrode. 


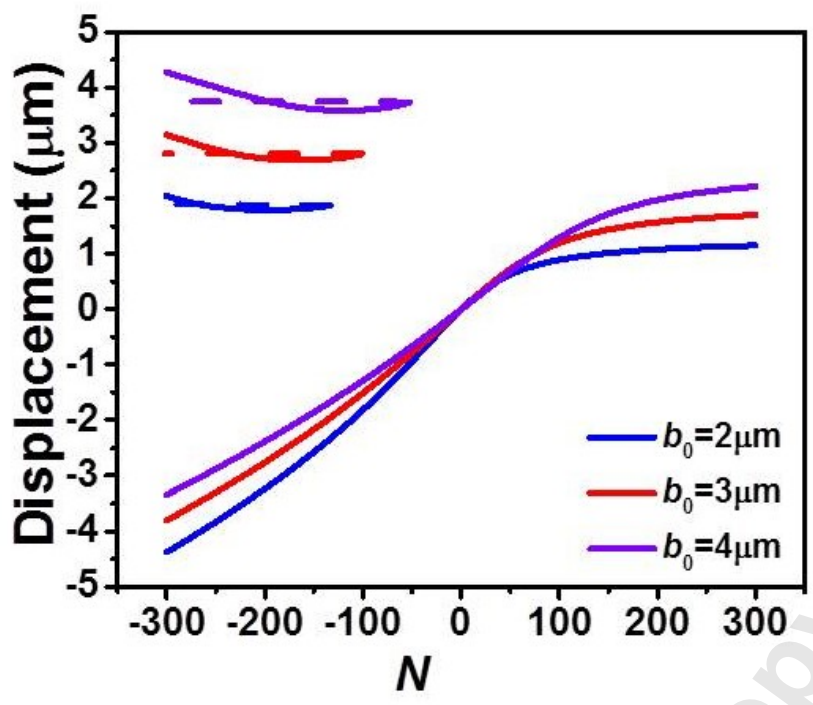

(a)

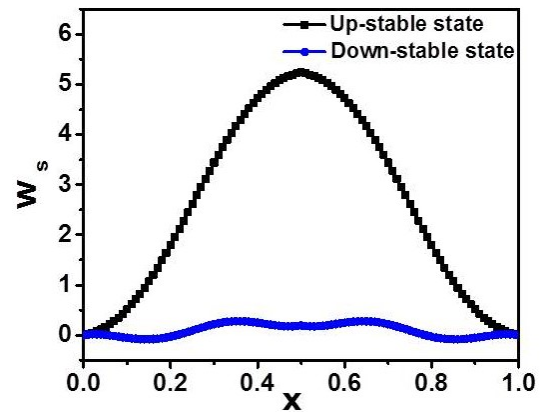

(b)

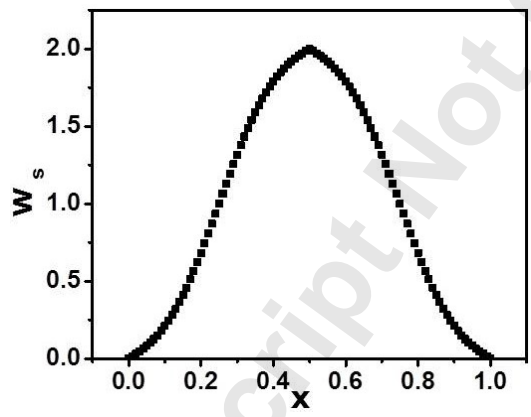

(c)

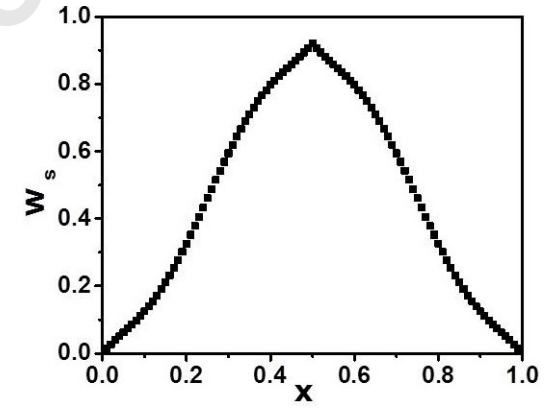

(d)

Fig. 2. (a)Variation of the static deflection of the V-shaped micro-beam versus the normalized axial load $(N)$ for zero DC load $\left(V_{D C}=0 \mathrm{~V}\right)$ and assuming various values of initial rise $b_{0}$ (solid: stable, dashed: unstable). The static deflection of the micro-beam with $V_{D C}=0 \mathrm{~V}$ at initial rise $b_{0}=2 \mu \mathrm{m}$ for (b) $\mathrm{N}=-200$, (c) $\mathrm{N}=0$, and (d) $\mathrm{N}=200$.

Subsequently, the effect of the normalized axial load on the maximum static of the V-shaped structure at its midpoint for several values of the micro-beam initial rise and assuming zero DC load are shown in Fig. 2a. It can be clearly seen that as a positive axial force (tensile $N$ ) increases, the V-shaped micro-beam deflects positively toward the electrode. This drift lasts till the $\mathrm{V}$-shaped micro-beam's curvature reduces leading to a slightly conventional shallow arched beam shape, Fig. $2 d$ compared to the reference initial V-shape shown in Fig. 2c. Here, the structure's displacement reaches a limit and it is no longer increasing with any further axial load. A saturation near $N \approx 300$ is registered for almost all assumed initial rise values Fig. 2a. In contrast, assuming a negative axial force (compressive $N$ ) pushes the micro-beam up, i.e. negative relative displacement, and as the compressive load is further increased, the microbeam static profile continues to increase till attaining a threshold value, after which two stable equilibrium solutions emerge and coexist: a lower solution representing the upward position of the micro-beam and an upper solution corresponding to the snapped V-shaped profile, Fig.2b. \ 
In Fig. 3a, the effect of the initial rise on the maximum static deflection of the V-shaped microbeam with the DC load and assuming $N=0$ is shown. The figure shows that for the cases of $b_{o}=2$ $\mu m$, the micro-beam undergoes a continuous snap-through first and then pull-in. However, for the case of $b_{o}=3 \mu \mathrm{m}$, the $\mathrm{V}$-shaped micro-beam snaps-through first and pulls-in while increasing $V_{D C}$ (see static profiles). Yet, increasing further the initial rise of the micro-beam to $4 \mu \mathrm{m}$, the $\mathrm{V}$ shaped micro-beam undergoes an immediate pull-in when increasing $V_{D C}$ without any possibility to snap-through. It is worth mentioning that, for this latter case, there is a band of $V_{D C}$, where two stable solutions are co-existing: one original stable solution (concaved up configuration, see static profile) and a snapped (concaved down configuration). Fig. 3a also demonstrates that the snap-through voltage growths and the pull-in voltage declines when growing the initial rise value of the V-shaped structure. This shows that the stiffness of the micro-structure upsurges before snap-through and then reduces in the symmetric position with the increase of $b_{o}$.

A deep explanation of the influence of the $V_{D C}$ with $N=0$ on the static deflection of the microbeam is given in Figs. 3 (b)-5(f). As we mention above, a positive $w_{s}$ indicates upward relative displacement with respect to the initial V-shape of micro-beam. Applying a DC voltage $\left(V_{D C}\right)$ between the V-shaped micro-beams and the stationary electrodes induces an attractive electrostatic force, which deflects the movable micro-beams towards the stationary electrodes. Figs. 3(b) and 3(c) show the results for the case of $b_{o}=2 \mu \mathrm{m}$. It is found that for a low voltage $V_{D C}=50 \mathrm{~V}$, the micro-beam shows a small deflection, see Fig. $3 \mathrm{~b}$. On the other hand, increasing $V_{D C}$ to $70 \mathrm{~V}$, the result shows significantly large deformation of the micro-beam, Fig. 3(c). As noted, the voltage in this case is near pull-in. Figs. 3(d) and 3(e) show that for $b_{o}=3 \mu \mathrm{m}$, the micro-beam indicates two stable states around $50 \mathrm{~V}$ and pull-in behavior around $70 \mathrm{~V}$ with a negative deflection toward the electrode. However, for $b_{o}=4 \mu \mathrm{m}$, the result shows remarkable deflection in the opposite direction, Figs. 3(f). 


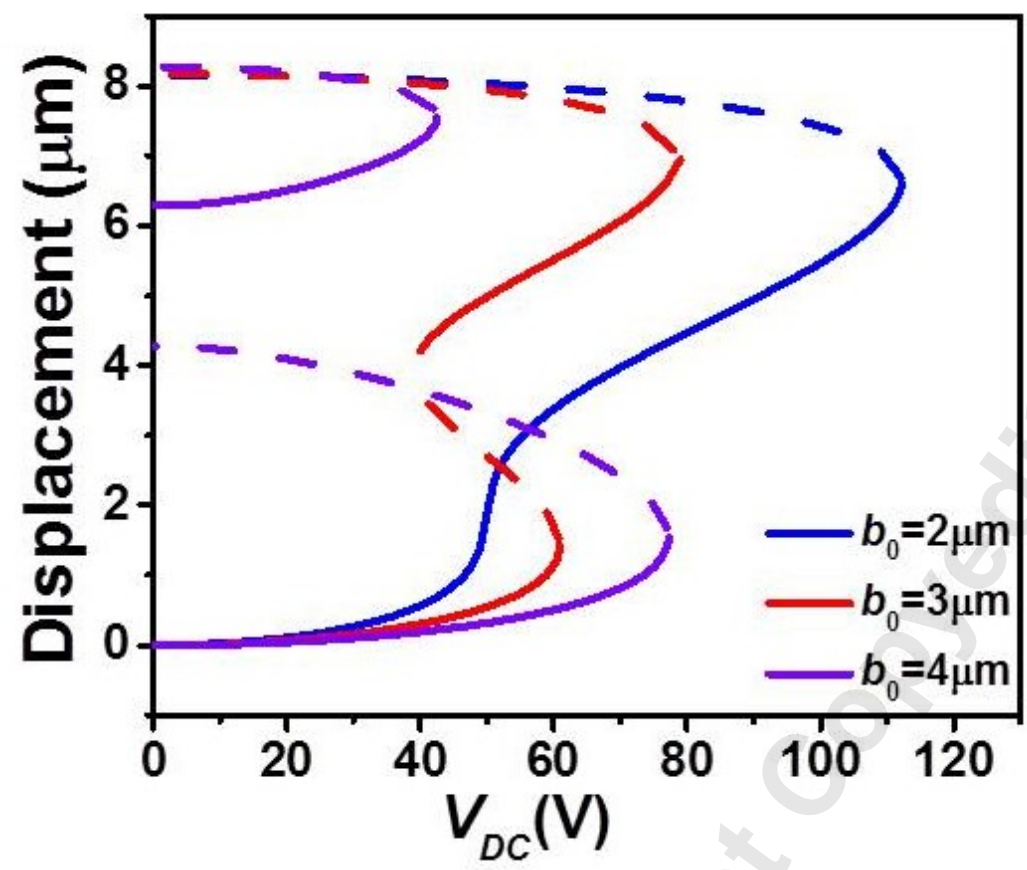

(a)

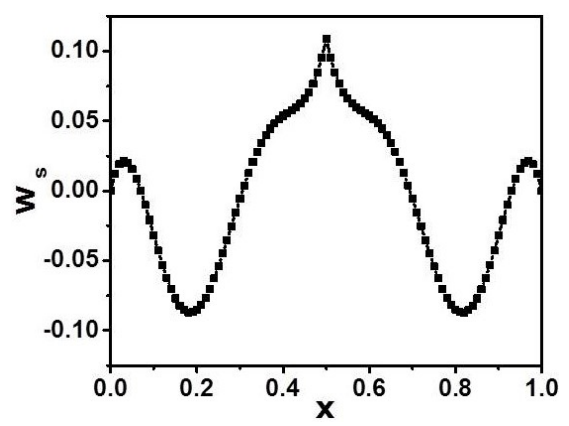

(b)

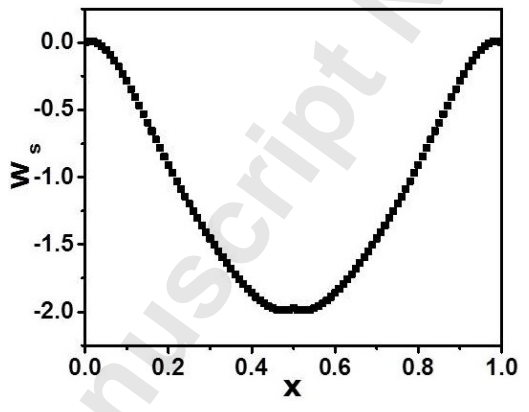

(c)

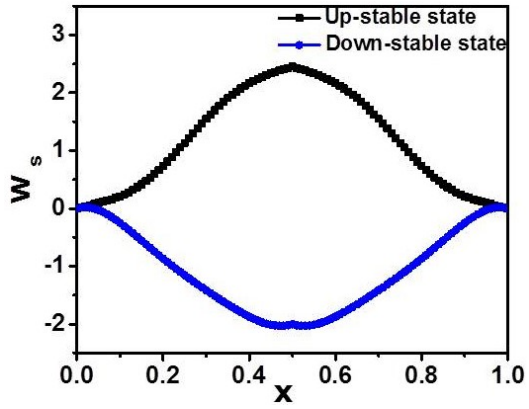

(d)

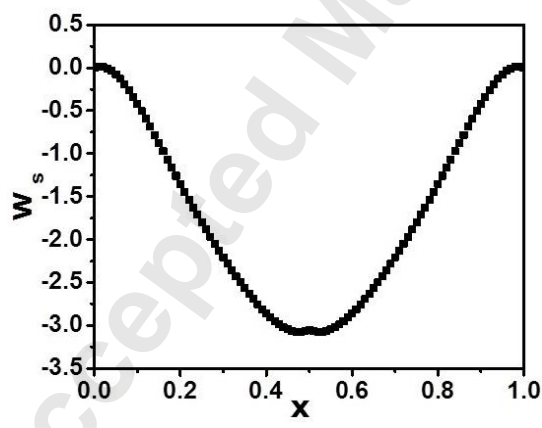

(e)

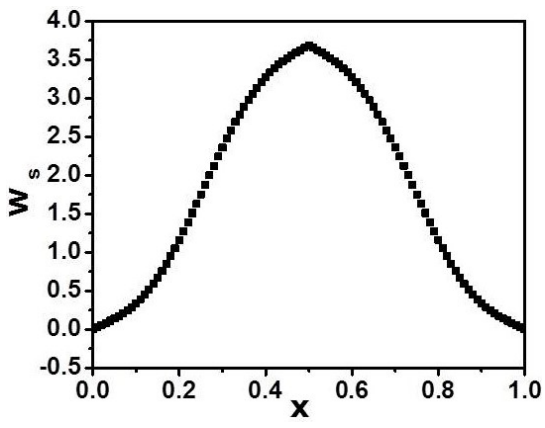

(f)

Fig. 3. (a)Variation of the static deflection of the $\mathrm{V}$-shaped micro-beam with $V_{D C}$ for various values of initial rise $b_{0}$ and for $N=0$ (solid: stable, dashed: unstable). (b)-(f) The static deflection of the micro-beam at $N=0$ under various $V_{D C}$ and while changing $b_{0}$. (b) $V_{D C}=50 \mathrm{~V}, b_{0}=2 \mu \mathrm{m}$, (c) $V_{D C}=70 \mathrm{~V}, b_{0}=2 \mu \mathrm{m}$, (d) $V_{D C}=50 \mathrm{~V}, b_{0}=3 \mu \mathrm{m}$, (e) $V_{D C}=70$ $\mathrm{V}, b_{0}=3 \mu \mathrm{m}$, and (f) $V_{D C}=50 \mathrm{~V}, b_{0}=4 \mu \mathrm{m}$. 
Next, the effect of the DC attractive load on the V-shaped micro-beam deflection and for various values of axial forces and initial rises are highlighted in Figs. 4a and 4b. In Fig. 4a, for an initial rise $b_{o}=3 \mu \mathrm{m}$ and assuming a normalized tensile and compressive axial forces, $N=20$ and $N=-20$ respectively, applying a compressive axial force tends to slightly push the micro-structure up, which can be noted by the negative deflections for minor values of DC load. Then, the DC force pushed the micro-beam in the downward direction and thus the maximum static deflection increases with the DC load until a snap-through occurs, where the micro-beam switches from a concaved-up to a concaved-down state. At the snapped state, the V-shaped structure become very sensitive to any change in the DC load and any increase of this voltage lead eventually to the pull-in voltage. The same figure, Fig. 4a, shows that a tensile load increases the pull-in voltage and decreases the snap-through voltage, however, a compressive load does the opposite and a wider hysteresis band (co-existence of two stable solutions) is observed in this case.

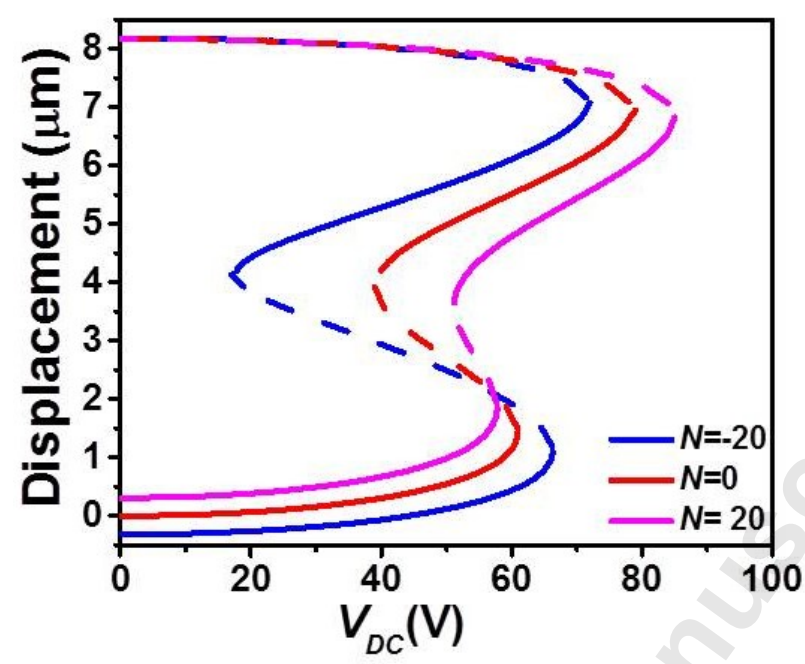

(a)

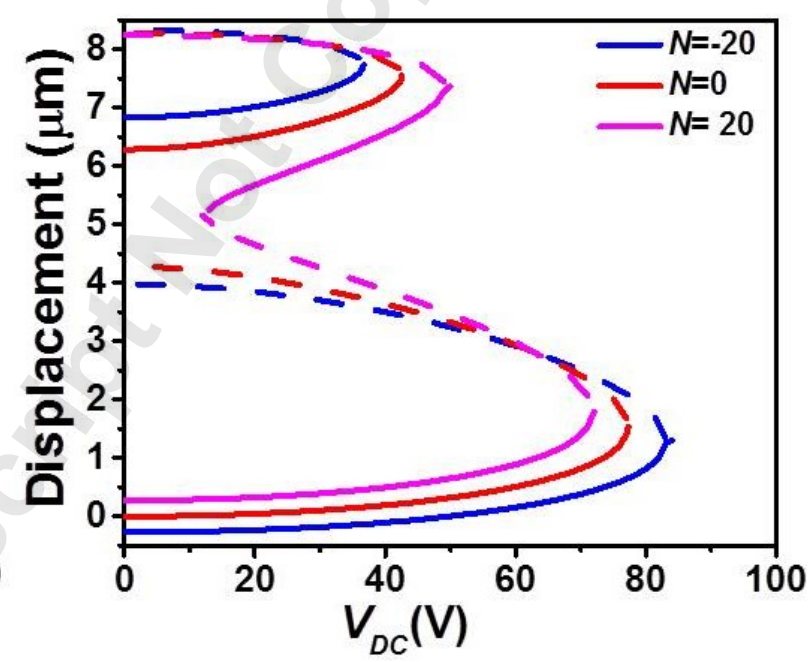

(b)

Fig. 4. Variation of the static deflection of the $\mathrm{V}$-shaped micro-beam with $V_{D C}$ at $\mathrm{N}=-20,0$, and 20 for: (a) $b_{0}=3$ $\mu \mathrm{m}$ and (b) $b_{0}=4 \mu \mathrm{m}$ (solid: stable, dashed: unstable).

For the case of higher initial rise, $b_{o}=4 \mu m$, Fig. $4 \mathrm{~b}$ shows similar trend, however here the microbeam static defection shows an inevitable pull-in without a possibility of passage to a snapped configuration. The same figure, Fig. 4b, shows also that a tensile load decreases the pull-in voltage and a compressive load just shows completely the opposite trend. 


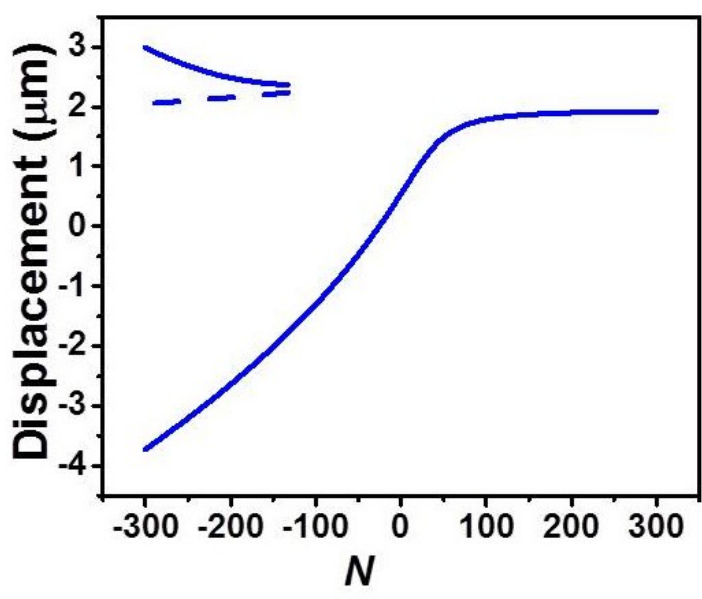

(a) $V_{\mathrm{DC}}=50 \mathrm{~V}$

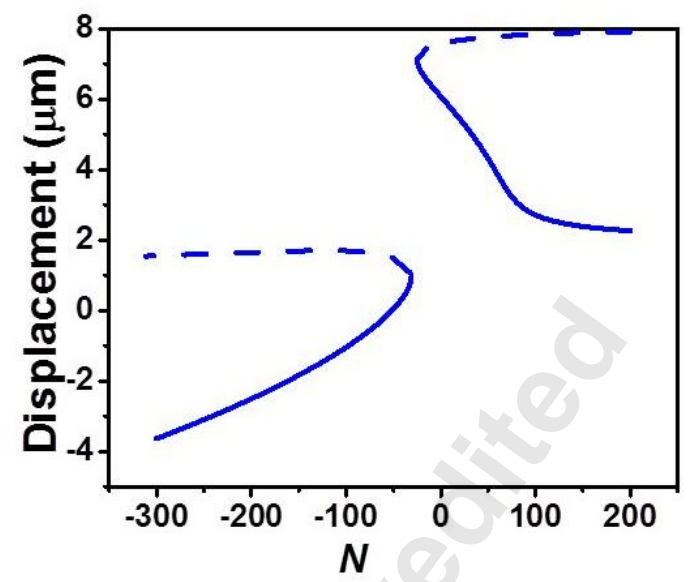

(b) $V_{\mathrm{DC}}=70 \mathrm{~V}$

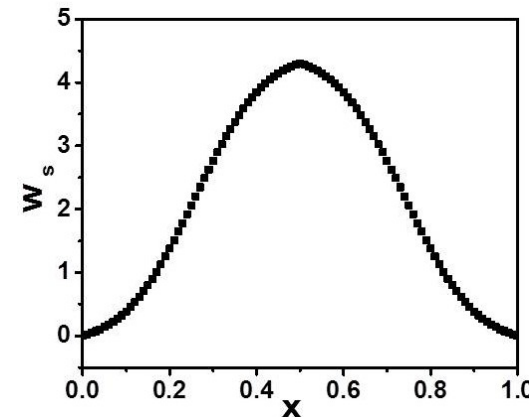

(c)

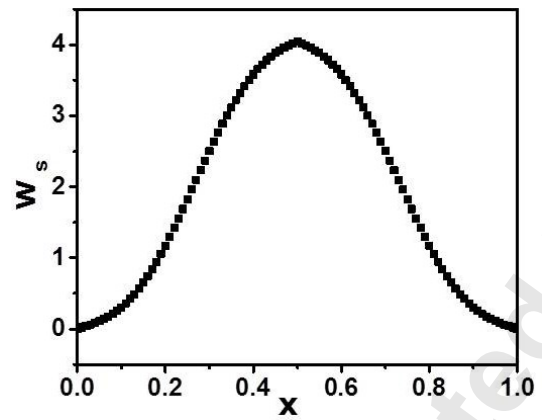

(f)

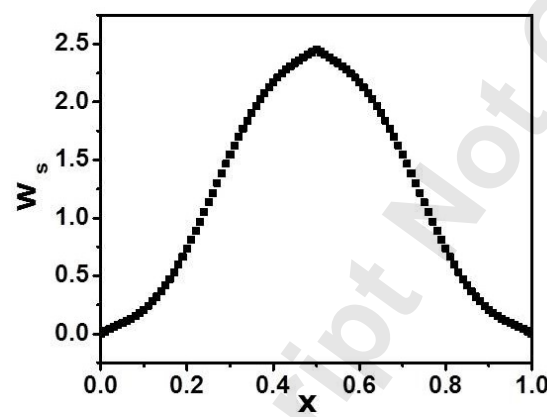

(d)

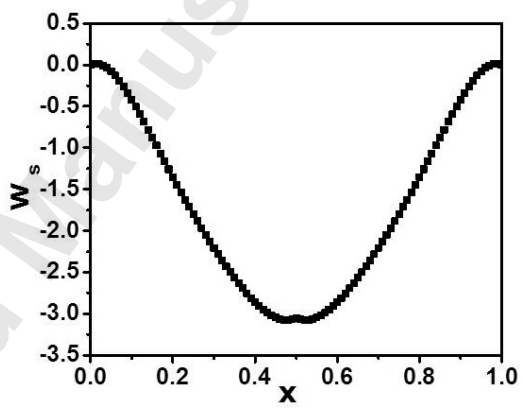

$(\mathrm{g})$

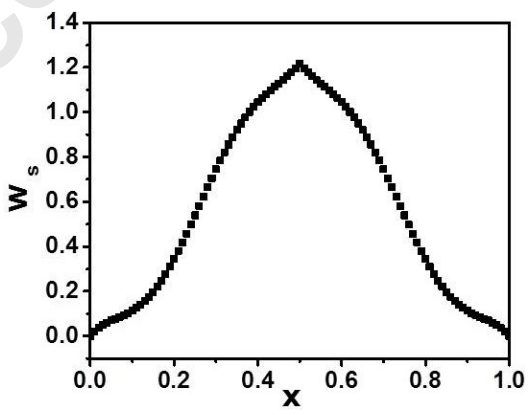

(e)

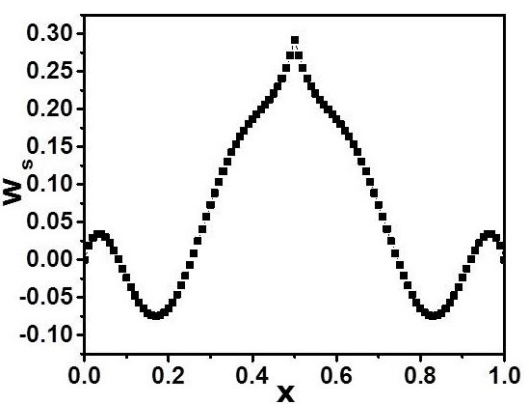

(h)

Fig. 5. Variation of the static deflection of the $\mathrm{V}$-shaped micro-beam versus the normalized axial load $N$ at initial rise $b_{0}=3 \mu \mathrm{m}$ for (a) $V_{D C}=50 \mathrm{~V}$ and (b) $V_{D C}=70 \mathrm{~V}$ (solid: stable, dashed: unstable). The static deflection of the micro-beam at (c)-(e) $V_{D C}=50 \mathrm{~V}$ and (f)-(h) $V_{D C}=70 \mathrm{~V}$ under various $N$ for (c,f) $N=-100,(\mathrm{~d}, \mathrm{~g}) N=0$, and (e,h) $N$ $=-100$.

Next, the static behavior of the V-shaped micro-beam of an initial rise of $b_{o}=3 \mu \mathrm{m}$ is examined with the axial force and assuming two values of the electrostatic loads, Fig. 5. Indeed, Figs. 5a and $5 \mathrm{~b}$ show the results for relatively small $\left(V_{D C}=50 \mathrm{~V}\right)$ and large voltage $\left(V_{D C}=70 \mathrm{~V}\right)$ loads, respectively. Furthermore, Fig. 5a appears comparable to that in Fig. 2a. This is reasonable, since a relatively small DC load causes very small displacement without changing qualitatively the overall static behavior of the V-shaped micro-beam, Figs. 5c-5e. Contrarywise, Fig. 5b shows a 
situation where the DC load amplitude is causing the V-shaped micro-beam to snap closer to its actuating electrodes at zero axial force, as shown in Fig. 5g. At this state, an assumed tensile axial force $(N=100)$ reduces the curved profile of the $\mathrm{V}$-shaped structure causing it to move at a concaved-up state, Fig. 5h. Increasing further the axial load positively results into a reduction in the curvature until reaching an almost concaved-up position. Conversely, assuming compressive (negative) axial loads upsurge the static defection of the V-shaped micro-beam leading to an increase in the total deflection upward away from the actuating electrode, Fig. 5f. At a specific value of compressive load where two new solutions co-exist: a stable upward solution and an unstable downward state. This is mainly called a bifurcation state.

\section{Dynamic Analysis: Eigenvalue Problem}

To demonstrate the validity of the analytical/numerical model, a comparison with experimental data for a case study is conducted. An image of the tested V-shaped micro-beam is shown in Fig. 6a. The micro-beam is fabricated from silicon-on-insulator (SOI) wafer from MEMSCAP [30]. The micro-beam is of length $(L) 500 \mu \mathrm{m}$, thickness $(h) 2 \mu \mathrm{m}$, and initial rise $\left(b_{0}\right) 5 \mu \mathrm{m}$. As shown in Fig. 6a, the electrostatic voltage $\mathrm{V}_{\mathrm{DC}}$ is applied between the anchors of the micro-beam and the upper electrodes.

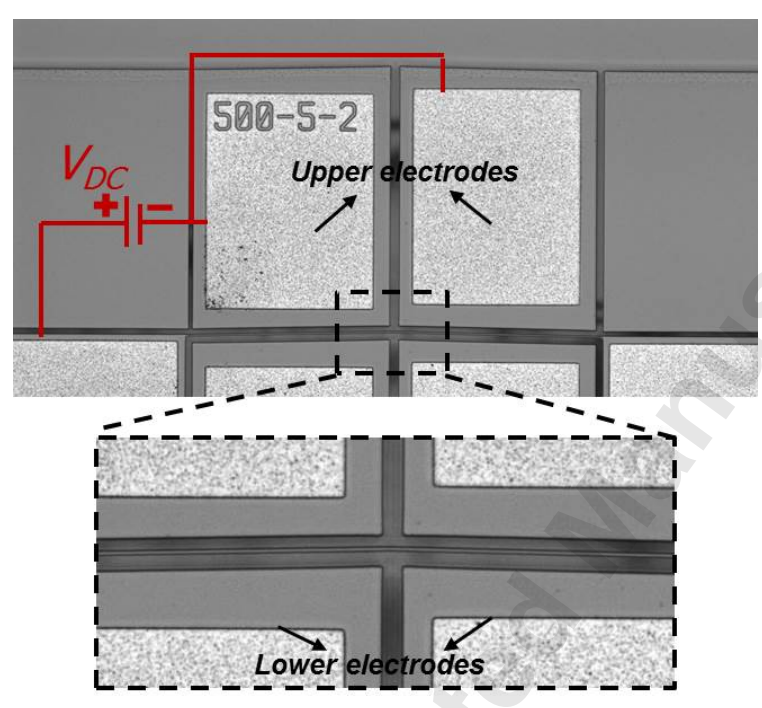

(a)

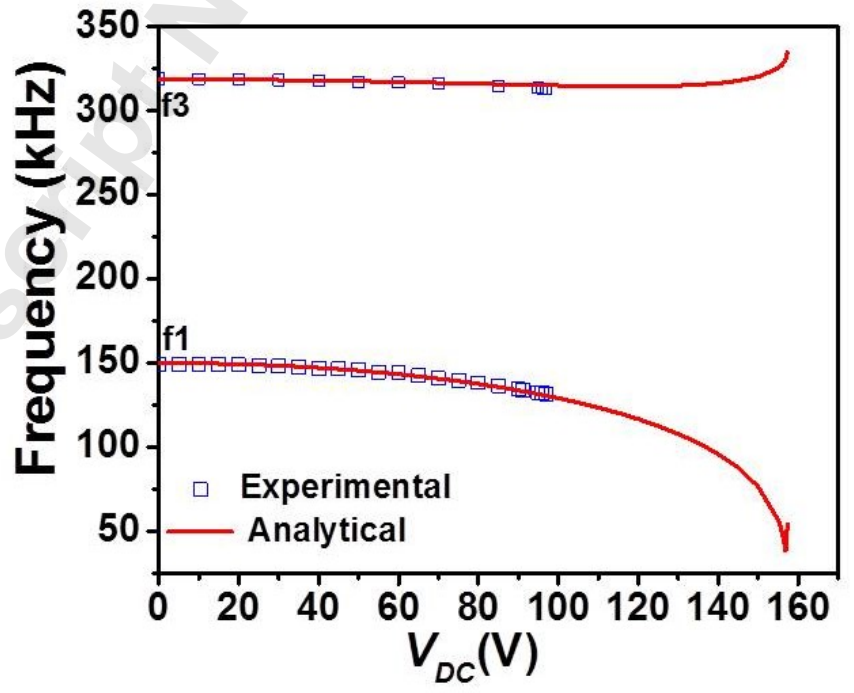

(b)

Fig. 6. (a) An optical top-view image of the V-shaped micro-beam with a schematic of the actuation mechanism. (b) Variation of the first two symmetric resonance frequencies $\left(\mathrm{f}_{1}\right.$ and $\left.\mathrm{f}_{3}\right)$ versus $V_{D C}$ obtained experimentally (scatters) and theoretically (lines).

Here we classify two types of stationary in-plane electrodes. Lower electrodes refer to in-plane electrodes placed inward of the curvature (inclination) of the structure; and upper electrodes refer to electrodes placed outward of the curvature; as seen in Fig 6a. The variation of the frequencies is measured with optical sensing (laser) when the micro-beam is subjected to an electrostatic white noise signal superimposed to a DC bias provided by the MSA. Also, a separate DC 
electrostatic voltage source VDC is provided using programmable DC power supplies, which induces an electrostatic force between the movable micro-beam and the upper electrodes. The micro-resonator is tested under ambient pressure and room temperature. Fig. $6 \mathrm{~b}$ shows the results of the variation in the first two symmetric $\left(f_{1}\right.$ and $\left.f_{3}\right)$ resonance frequencies. Good agreement is noted between the analytical model results and experimental data.

Fig. 7 shows the variation of the fundamental, Fig. 7a, and third, Fig. 7b, natural frequencies with the axial loads for three cases of initial rise at zero electrostatic voltage. As discussed previously, for higher values of the initial rise, a positive axial force decreases the initial shape of the micro-beam toward an almost straight configuration. This unstiffens the $\mathrm{V}$-shaped structure reducing its fundamental natural frequency. Once the micro-beam reaches an almost flat configuration, it behaves like a straight beam and gains stiffness with the increase in $N$, thereby increasing its natural frequency. On the other hand, any compressive axial force broadens the micro-beam curvature reinforcing its overall stiffness, and thus increasing its natural frequency. This trend continues till passing the snap bifurcation where the fundamental frequency drops to zero. After this bifurcation, and following the concaved-down state, the natural frequencies increase with the increased of the compressive axial load.

Furthermore, Figs. $8 \mathrm{a}$ and $8 \mathrm{~b}$ demonstrate the effect of the initial rise and DC load on the fundamental and third natural frequencies of the investigated V-shaped microbeam. For several values of $b_{0}$, the fundamental frequency decreases monotonically to zero before reaching the snap-through instability, after which the same frequency shows an increase followed by another drop to zero near when reaching the pull-in instability. These trends are mainly attributed to the softening (of quadratic type) effect of both the initial shape of the structure and the actuating softening electric load. After the snap-through, a hardening behavior (mainly attributed to the beam cubic mid-plane stretching), is dominating the above-mentioned softening nonlinearities for certain range before reaching near the pull-in where the electric forces are mainly dominant.

Therefore, in certain regions, the fundamental natural frequency increases just after snap-through while increasing the DC voltage. Then, near pull-in, it drops again to zero. Besides, the third natural frequency, Fig. 8b, undertakes a decrease in their initial state before the snap-through instability, and then starts a constant increase right after the snap-through instability to pull-in, except for the case of higher, $b_{o}=4 \mu \mathrm{m}$, where the micro-structure inevitably goes to pull-in. 


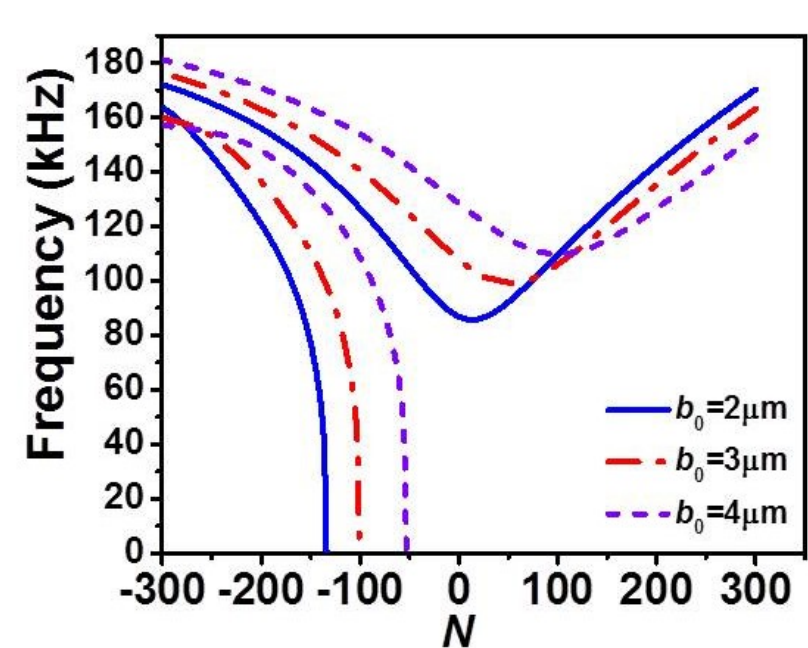

(a)

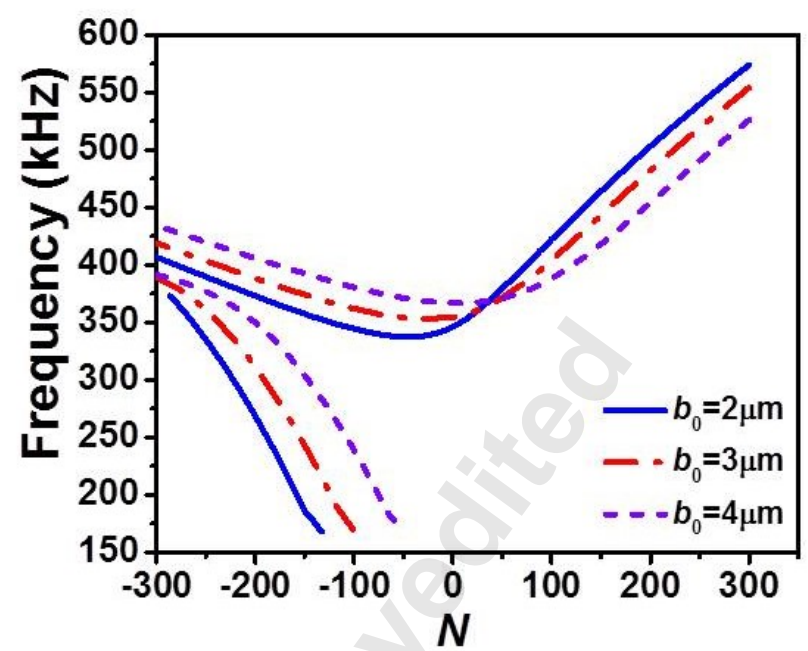

(b)

Fig. 7. Variation of the (a) first and (b) third natural frequencies versus $N$ for $V_{D C}=0 \mathrm{~V}$ and for various values of initial rise $b_{0}$.

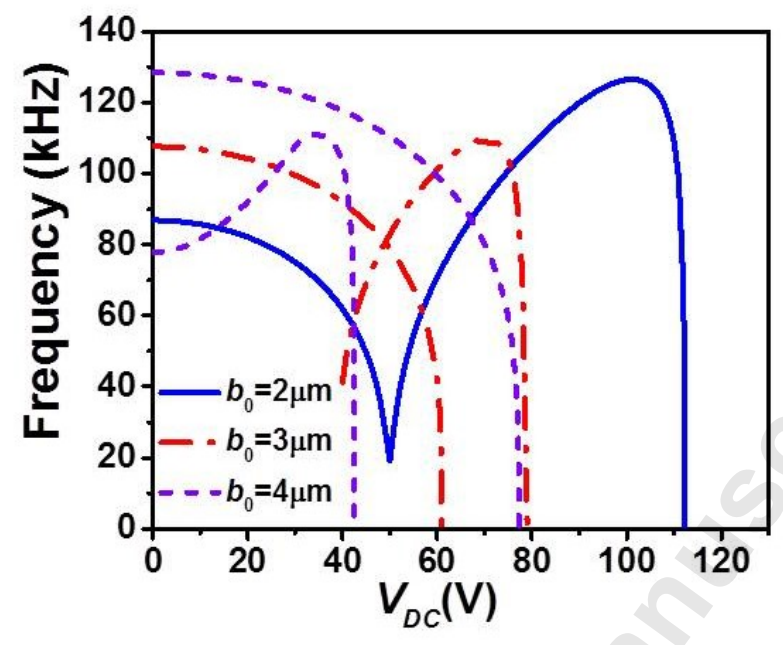

(a)

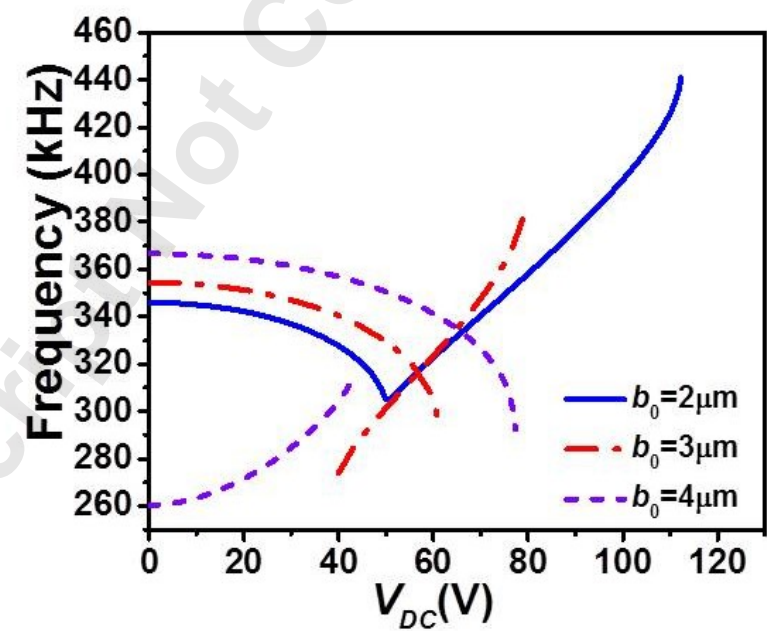

(b)

Fig. 8. Variation of (a) the first and (b) the third natural frequencies of the V-shaped micro-beam with $V_{D C}$ for various values of initial rise $b_{0}$ and $N=0$.

Figure 9 displays an analogous analysis to Fig. 7, nevertheless assuming DC voltages before the snap-through $\left(V_{D C}=50 \mathrm{~V}\right)$ and beyond it $\left(V_{D C}=70 \mathrm{~V}\right)$. For $V_{D C}=50 \mathrm{~V}$, Figs. $9 \mathrm{a}$ and $9 \mathrm{~b}$, the structure is in the curved-up state assuming small initial displacement, and therefore the variation of the first and third natural frequencies are almost same as with the case of zero DC load, Fig. 7. Increasing further the DC load to $70 \mathrm{~V}$, Figs. 9c and 9c, the V-shaped structure becomes concaved-down with a strong influence from the electrostatic force. Therefore, any assumed positive axial force (tensile load) reduces the curvature into a straight configuration, thus softening the micro-structure and reducing its natural frequencies. When the structure attains a nearly straight shape, it gains some stiffness with $N$, resulting into an increase in both frequencies. 
Nevertheless, assuming a compressive axial load, the V-shaped structure, initially at the concaved-down position, is strapped further downward in the direction of the lower actuating electrodes increasing accordingly the softening effect of the electrostatic force. This decrease both natural frequencies, where the fundamental one reaches zero ultimately at the pull-in instability. However, since this also represents a hysteresis region, another stable solution is coexisting which is corresponding to the concaved-up shape of the micro-beam. Considering this state, any increase in the compressive load leads to an increase in the natural frequencies.

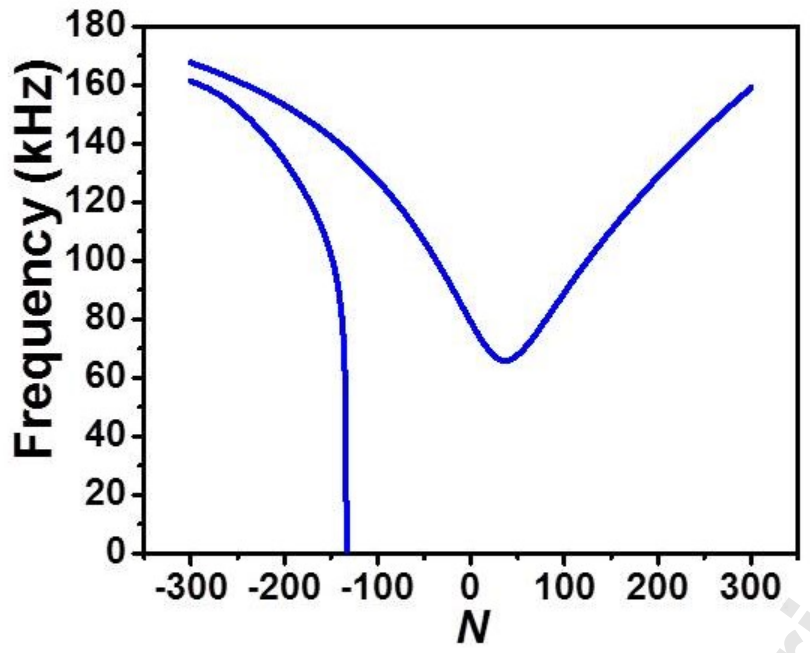

(a)

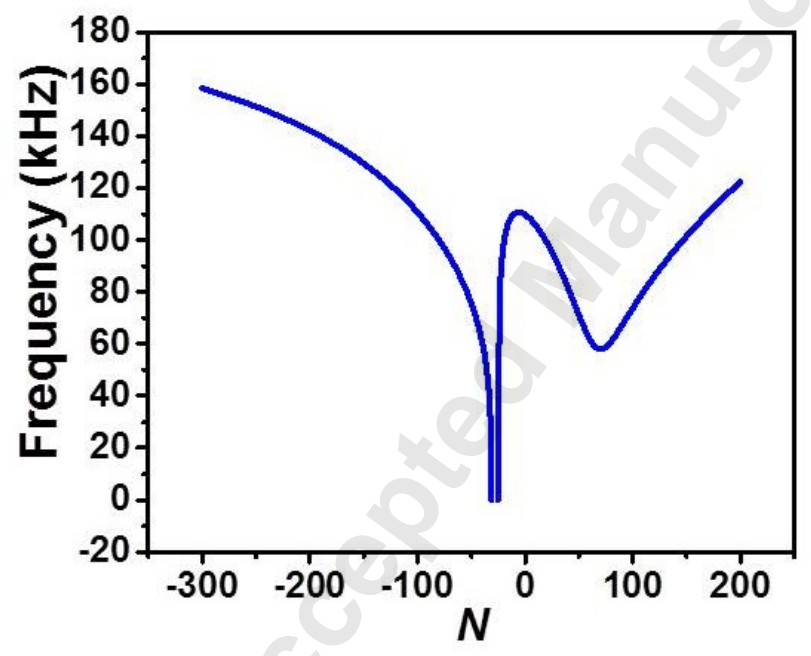

(c)

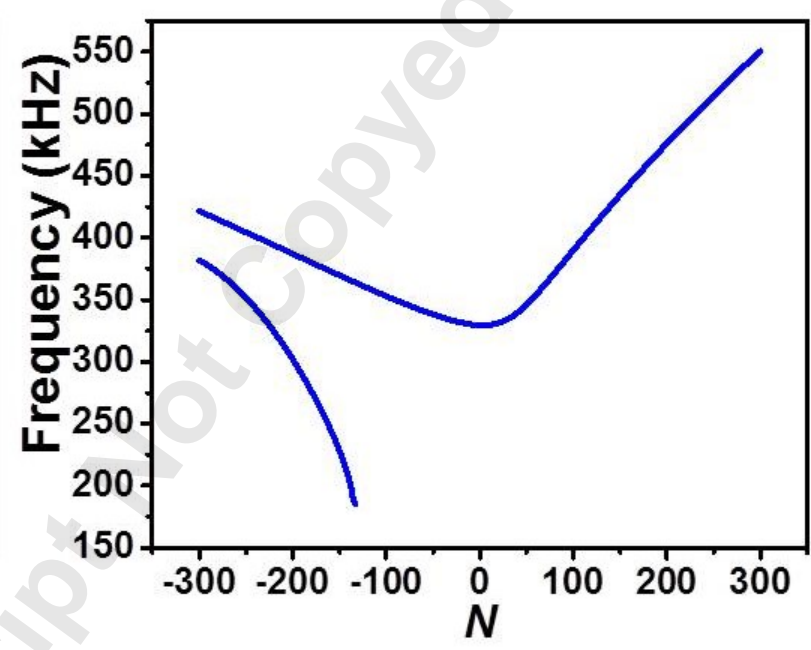

(b)

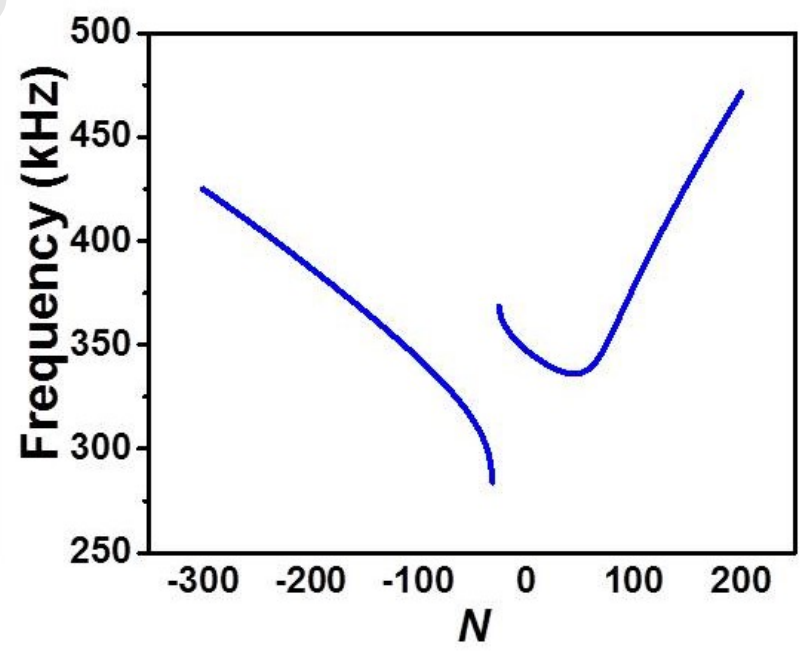

(d)

Fig. 9. Variation of the (a,c) first and (b,d) third natural frequencies versus $N$ at initial rise $b_{0}=3 \mu \mathrm{m}$ for $(\mathrm{a}, \mathrm{b}) V_{D C}=$ $50 \mathrm{~V}$ and (c,d) $V_{D C}=70 \mathrm{~V}$. Variation of (a) the static deflection and (B) the first natural frequencies of the Arcshaped micro-beam versus the normalized axial load $N$ at initial rise $b_{0}=3 \mu \mathrm{m}$ for $V_{D C}=50 \mathrm{~V}$ and (b70 V (solid: stable, dashed: unstable). The static deflection of the micro-beam at (c)-(e) $V_{D C}=50 \mathrm{~V}$ and (f)-(h) $V_{D C}=70 \mathrm{~V}$ under various $N$ for (c,f) $N=-100,(\mathrm{~d}, \mathrm{~g}) N=0$, and $(\mathrm{e}, \mathrm{h}) N=-100$. 
Figs. 10 and 11 demonstrate the fundamental and third natural frequencies variation with the DC attractive forces and assuming various states of axial loads for two values of the initial rise, $b_{o}=3$ $\mu m$ and $b_{o}=4 \mu m$, respectively. Both figures show that increasing both the DC voltage and the axial load reduce the natural frequencies. This remains until the micro-beam entirely loses its stability near snap-through for the case of $b_{o}=3 \mu \mathrm{m}$ and at the pull-in instability for the case of $b_{o}=4 \mu \mathrm{m}$. For the case of $b_{o}=3 \mu \mathrm{m}$, and beyond snap-through, the micro-beam is at the concaveddown configuration, both frequencies increase and the fundamental one starts to decrease and goes to zero near the pull-in instability. For both cases, any assumed compressive and tensile axial forces upsurge and reduces the hysteresis band, respectively, as shown in Figs. 10 and 11.

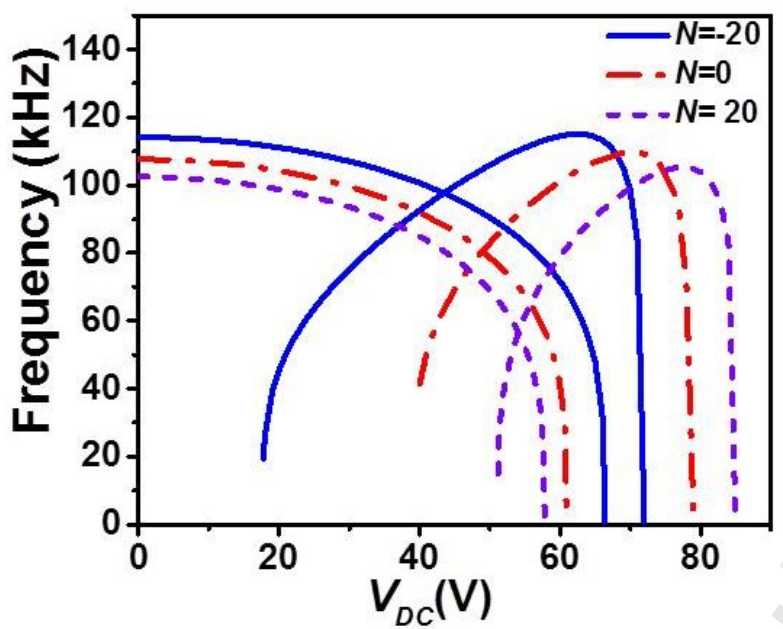

(a)

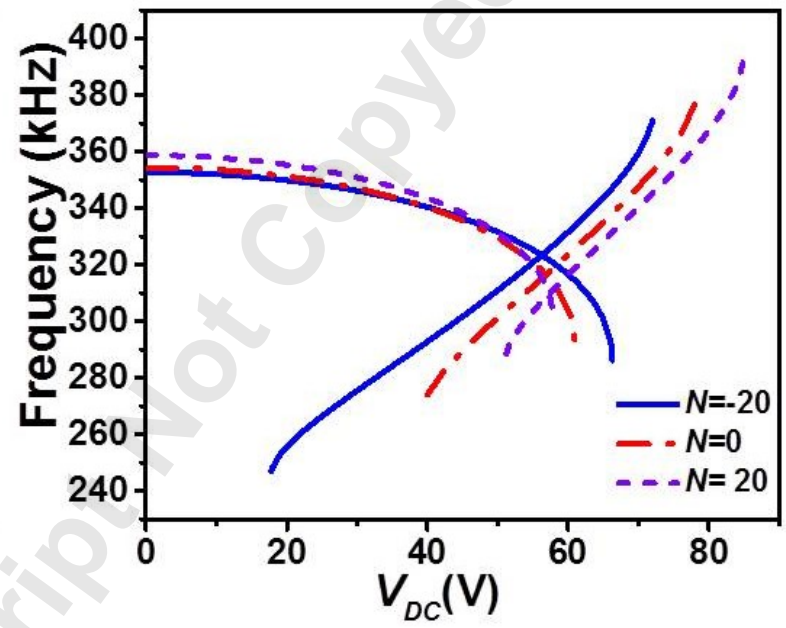

(b)

Fig. 10. Variation of (a) the first and (b) the third natural frequencies of the $\mathrm{V}$-shaped micro-beam with $V_{D C}$ at $\mathrm{N}=-$ 20,0 , and 20 for $b_{0}=3 \mu \mathrm{m}$.

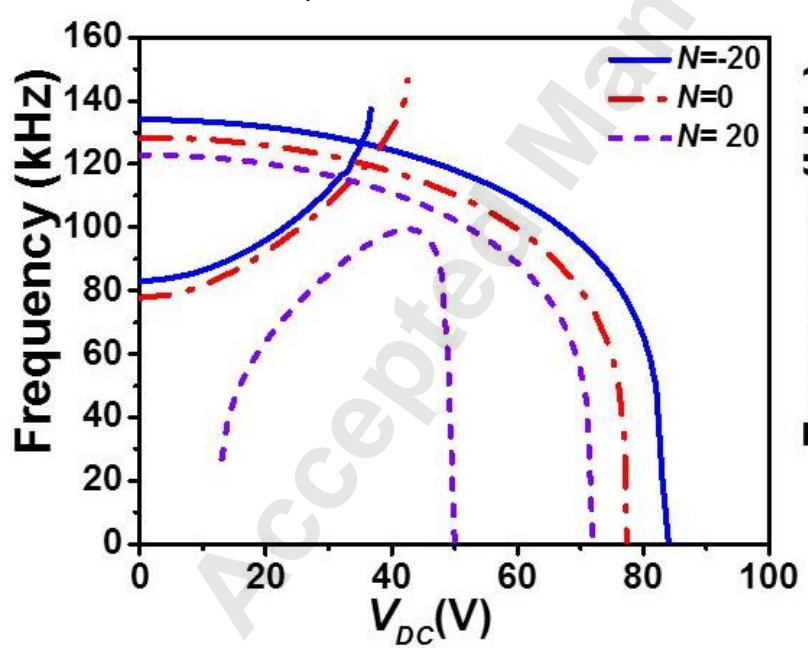

(a)

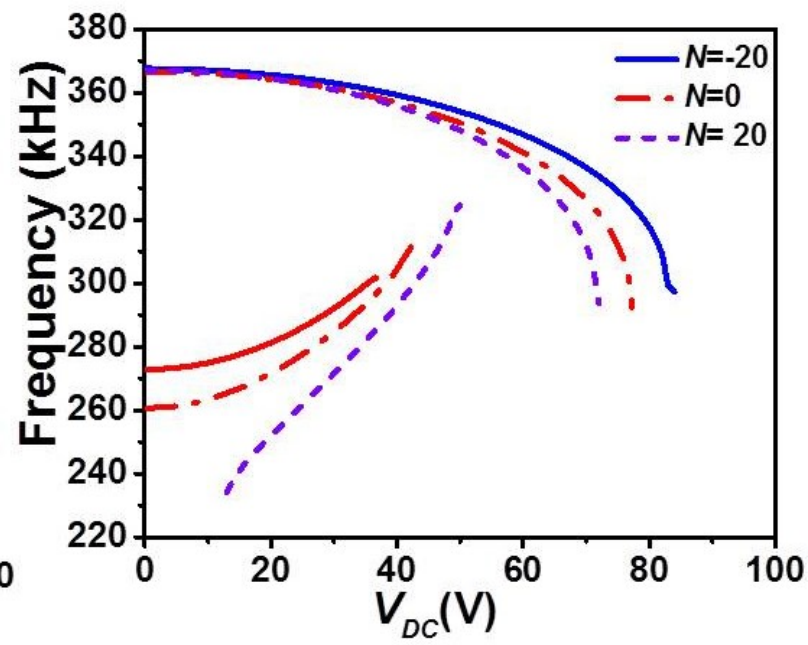

(b)

Fig. 11. Variation of the (a) first and (b) third natural frequencies of the $\mathrm{V}$-shaped micro-beam with $V_{D C}$ at $\mathrm{N}=-20$, 0 , and 20 for $b_{0}=4 \mu \mathrm{m}$. 
Finally, we aim to study the effect of the initial shape on the static and dynamic behavior of the micro-resonator. For that we consider an Arc-shaped micro-beam with the same geometrical parameters of the V-shaped micro-beam of table 1 and with an initial rise of $b 0=3 \mu \mathrm{m}$. According to the obtained results of Figs. 2a, 5a and 12a, the V-and Arc-shaped actuators have almost the same output displacement for the lower stable branch but the V-shaped arrangement do possess better hysteric behavior, when comparing both upper stable and unstable branches. Consequently, one can notice the same trend for the variation of the fundamental frequency for the V-shaped (Figs. 7a and 9a) and the Arc-shaped outline (Fig. 12b). Although the Arch-shaped case is showing better tunability in the stable upper branch, the V-shaped beam is showing better hysteretic behavior allowing even more frequency tunability moving from one side of the microbeam to the other.

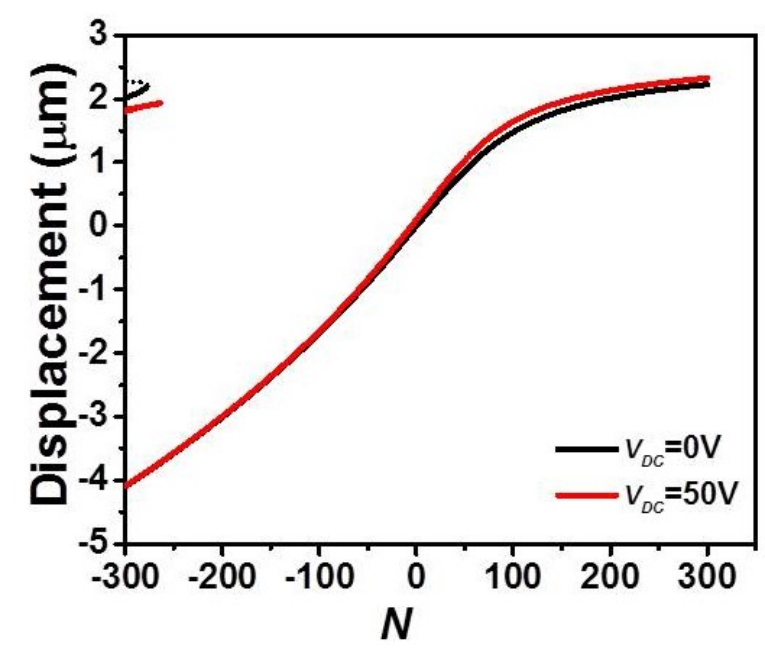

(c)

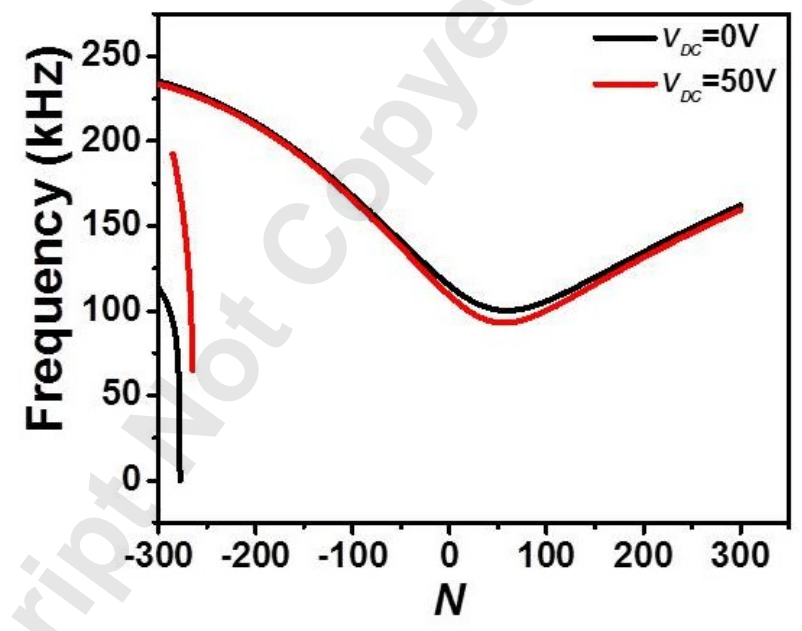

(d)

Fig. 12. Variation of (a) the static deflection and (b) the first natural frequency of the Arc-shaped micro-beam versus the normalized axial load $N$ at initial rise $b_{0}=3 \mu \mathrm{m}$ for $V_{D C}=0 \mathrm{~V}$ and $50 \mathrm{~V}$ (solid: stable).

\section{Conclusions}

Numerically and Experimental investigations into the statics and dynamics (mainly the natural frequencies) of clamped-clamped electrostatically tuned V-shaped micro-beams were investigated. Both the pull-in and snap-through behaviors under the both effects of the DC attractive forces superimposed to compressive and tensile axial loads were examined. It was found that that the axial forces have significant effect on the behavior of such V-shaped structures resulting into a noticeable increase and/or reduction in the overall effective stiffness of the micro-beam and, therefore, an increase and/or decrease in its resulting fundamental natural frequency. The increase or decrease was shown to mainly depend on the values of the attractive DC force, the initial deformation of the $\mathrm{V}$-shaped structure and whether the axial loads are of tensile or compressive nature. Furthermore, some multi-solutions (hysteresis) domains were computed resulting into multi-state scenarios, particularly for the case of compressive axial loads. Likewise, results showed promising opportunities of the exploitation of axial loads to tune 
the fundamental natural frequency of these $\mathrm{V}$-shaped structures, when used as resonators, over a wide range of frequencies (lower and/or higher values). This offers the possibility to improve the properties of this micro-structure for applications in need of high sensitivity, and represents a more attractive alternative of straight and even bell-shaped microbeams.

\section{Acknowledgment}

This research has been supported through King Abdullah University of Science and Technology (KAUST) fund.

\section{References}

[1] Alcheikh, Nouha, et al. "A highly sensitive and wide-range resonant magnetic micro-sensor based on a buckled micro-beam." Sensors and Actuators A: Physical (2021): 112768.

[2] Hasan, Mohammad H., Fadi M. Alsaleem, and Hassen M. Ouakad. "Novel threshold pressure sensors based on nonlinear dynamics of MEMS resonators." Journal of Micromechanics and Microengineering 28.6 (2018): 065007.

[3] Zamanzadeh, Mohammadreza, Ilgar Jafarsadeghi-Pournaki, and Hassen M. Ouakad. "A resonant pressure MEMS sensor based on levitation force excitation detection." Nonlinear Dynamics 100.2 (2020): 1105-1123.

[4] S. Yang, Q. Xu Q, A review on actuation and sensing techniques for MEMS-based microgrippers, Journal of Micro-Bio Robotics. 13(2017)1-4.

[5] A. Pimpin, I. Charoenbunyarit, W. Srituravanich, Material and performance characterization of Z-shaped nickel electrothermal micro-actuators, Sensors and Actuators A: Physical. 253(2017)49-58.

[6] X. L. Jia, J. Yang, S. Kitipornchai, C, W. Lim, Pull-in instability and free vibration of electrically actuated polySiGe graded micro-beams with a curved ground electrode, Applied Mathematical Modelling 36(2012)1875-84.

[7] M. Derakhshani, T. A. Berfield, Snap-through and mechanical strain analysis of a MEMS bistable vibration energy harvester, Shock and Vibration. (2019) 1-10, https://doi.org/10.1155/2019/6743676.

[8] G. M. Rebeiz, RF MEMS: theory, design, and technology. John Wiley \&amp; Sons; 2004 Feb 6.

[9] Z. Zhang, W. Zhang, Q. Wu, Y. Yu, X. Liu, and X. Zhang, Closed-form modelling and design analysis of Vand Z-shaped electrothermal microactuators,"Journal of Micromechanics and Microengineering. 27(2016) 015023.

[10] C. Guan, and Y. Zhu , An electrothermal microactuator with Z-shaped beams,Journal of micromechanics and microengineering. 20 (2010) 085014.

[11] M. M. Zand, The dynamic pull-in instability and snap-through behavior of initially curved microbeams, Mechanics of Advanced Materials and Structures. 19(2012)485-91.

[12] S. A. Tajalli, S. M. Tajalli SM, Wavelet based damage identification and dynamic pull-in instability analysis of electrostatically actuated coupled domain microsystems using generalized differential quadrature method.

Mechanical Systems and Signal Processing. 133(2019)106256.

[13] I. Z. Pane, T. Asano, Investigation on bistability and fabrication of bistable prestressed curved beam Japanese Journal of Applied Physics. 47 (2008)5291-5296.

[14] H. M. Ouakad HM, M. I. Younis, On using the dynamic snap-through motion of MEMS initially curved microbeams for filtering applications, Journal of Sound and Vibration. 333(2014)555-68.

[15] Alcheikh, Nouha, et al. "Static and dynamic actuations of clamped-clamped V-shaped micro-resonators under electrostatic forces." Mechanical Systems and Signal Processing 155 (2021): 107571.

[16] Alcheikh, Nouha, Hassen M. Ouakad, and Mohammad I. Younis. "Dynamics of V-Shaped Electrothermal MEMS-Based Resonators." Journal of Microelectromechanical Systems 29.5 (2020): 1372-1381.

[17] Zamanzadeh, Mohammadreza, Hassen M. Ouakad, and Saber Azizi. "Theoretical and experimental investigations of the primary and parametric resonances in repulsive force based MEMS actuators." Sensors and Actuators A: Physical 303 (2020): 111635.

[18] Hassena, Mohamed Amin Ben, et al. "2D electrostatic energy harvesting device using a single shallow arched microbeam." International Journal of Non-Linear Mechanics 132 (2021): 103700.

[19] K. Song, H. Lee, Y. A.Cha, V-Shaped actuator utilizing electrostatic force, Actuators 7(2018) 30. Multidisciplinary Digital Publishing Institute.

[20] C. Goll, W. Bacher, B. Büstgens, D. Maas, W. Menz, W. K. Schomburg, Microvalves with bistable buckled polymer diaphragms, Journal of Micromechanics and Microengineering. 6 (1996)77-79. 
[21] M. A. Hafiz, L. Kosuru, A. Ramini, K. N. Chappanda, M. I. Younis, In-plane MEMS shallow arch beam for mechanical memory, Micromachines. 7(2016)191.

[22] S. A. Alkharabsheh, M. I. Younis, Statics and dynamics of MEMS arches under axial forces, Journal of Vibration and Acoustics. 135(2013).

[23] L. Li, Z. J. Chew, Microactuators: design and technology. Smart sensors and MEMS Jan 1 (2018) $313-354$. Woodhead Publishing.

[24] N. Alcheikh N, . A. Tella, M. I. Younis, Adjustable static and dynamic actuation of clamped-guided beams using electrothermal axial loads. Sensors and Actuators A: Physical. 273(2018)19-29.

[25] N. Alcheikh , H. M. Ouakad, M. I. Younis, Dynamics of V-Shaped Electrothermal MEMS-Based Resonators. Journal of Microelectromechanical Systems. 29(2020):1372-81.

[26] X. Shen , X. Chen, Mechanical performance of a cascaded V-shaped electrothermal actuator, International Journal of Advanced Robotic Systems. 10(2013):379.

[27] E. T. Enikov, S. S. Kedar, K. V. Lazarov, Analytical model for analysis and design of V-shaped thermal microactuators, Journal of Microelectromechanical Systems. 14(2005)788-98.

[28] M. I.Younis, E. M. Abdel-Rahman, A. Nayfeh, A reduced-order model for electrically actuated microbeambased MEMS, Journal of Microelectromechanical systems. 12(2003):672-80.

[29] Younis M. I. MEMS Linear and Nonlinear Statics and Dynamics. (2011). New York: Springer.

[30] N. Alcheikh, A. Ramini, M. A. Hafiz, M. I. Younis, Tunable clamped-guided arch resonators using electrostatically induced axial loads. Micromachines. 28(2017)14. 